\title{
Stent-Graft Relining in a Patient with Acute Aortic Aneurysm and a Completely Migrated Endograft
}

Jayandiran Pillai ${ }^{1}$, BSc, MBBCh, FCS, CVS; Ceyhan Yazicioglu'ㄹ, BSc, BHScHon; Mahad Omar ${ }^{1}$, BMS, BScHon, BM; Martin G. Veller ${ }^{1}$, MBBCh, FCS, MMed

DOI: 10.21470/1678-9741-2017-0122

\section{Abstract}

Stent-graft migration and type I endoleaks are associated with a higher rate of reintervention and increased mortality and morbidity. This article describes a patient presented with an infrarenal aortic stent-graft which had migrated into the aortic sac with loss of all aortic neck attachment. The acutely expanding abdominal aortic aneurysm was treated by placing a second modular endograft within and above the migrated stentgraft. The patient returned $\mathbf{3 6}$ months later, with features of an acute myocardial infarction, severe bilateral lower limb ischemia, and renal failure. He was too ill for intervention and demised within 48 hours.

Keywords: Endovascular Procedures. Aortic Aneurysm, Abdominal. Stents/adverse effects. Blood Vessel Prosthesis/ adverse effects. Blood Vessel Prosthesis Implantation/adverse effects. Minimally Invasive Surgical Procedures/adverse effects.

\begin{tabular}{ll}
\hline Abbreviations, acronyms \& symbols \\
\hline AAA $\quad$ Abdominal aortic aneurysm \\
CT $\quad=$ Computed tomography \\
EVAR $\quad=$ Endovascular aneurysm repair \\
MI = Myocardial infarction \\
MG = Migrated stent-graft \\
SG = Second modular bifurcated endograft \\
SVS = Society for Vascular Surgery
\end{tabular}

\section{INTRODUCTION}

Endovascular aneurysm repair (EVAR) results in a lower 30-day mortality, compared to open repair ${ }^{[1,2]}$. However, the long term reintervention rate is high ${ }^{[1,2]}$. Migration and type I endoleaks are serious adverse events and indicate EVAR failure ${ }^{[3,4]}$. Distal migration of the main body, within a long infrarenal neck, may be treated by inserting an extender cuff, provided there is some overlap between the main body and neck ${ }^{[1,2,5]}$. More extensive forms of migration, when the main body is no longer contained within the neck (complete migration), may mandate conversion to open repair ${ }^{[2,3]}$. We will describe a medical highrisk patient presented with a completely migrated stent-graft

'Division of Vascular Surgery and Department of Surgery, University of the Witwatersrand, Johannesburg, South Africa.

This study was carried out at the University of the Witwatersrand, Johannesburg, South Africa.
(MG), abdominal pain, and a $10.5 \mathrm{~cm}$ infrarenal abdominal aortic aneurysm (AAA). The patient was treated by placing a second modular bifurcated endograft (SG) within and above the MG. Appropriate consent was obtained from the patient.

\section{CASE REPORT}

A 72-year old male presented with abdominal pain. Medical history included hypertension, diabetes and coronary artery disease. He had undergone endovascular repair, $6 \mathrm{~cm}$ AAA, five years before and had not returned for follow-up.

The computed tomography (CT) scan revealed a $10.5 \mathrm{~cm}$ infrarenal AAA and a totally migrated AneuRx ${ }^{\mathrm{TM}}$ stent-graft (Medtronic Inc. Minneapolis, USA). There was a complete displacement and angulation of the stent-graft's main body within the aneurysm sac (Figure 1). Both iliac components of the endograft were still maintained inside their respective iliac arteries (right limb as part of the main body and left limb as an intact extension). Due to his body mass index and comorbidities, we opted for endovascular stent-graft placement.

\section{Management Strategy and Planning}

The plan was to place a SG within and above the existing MG. The short SG'S (AnacondaTM Stent Graft System Vascutek Terumo, Inchinnan Renfrewshire, Glasgow, Scotland) main body

Correspondence Address:

Ceyhan Yazicioglu

58th Acacia Court, 6th Street - Linden - Johannesburg, South Africa

E-mail: c_yazicioglu@yahoo.com 


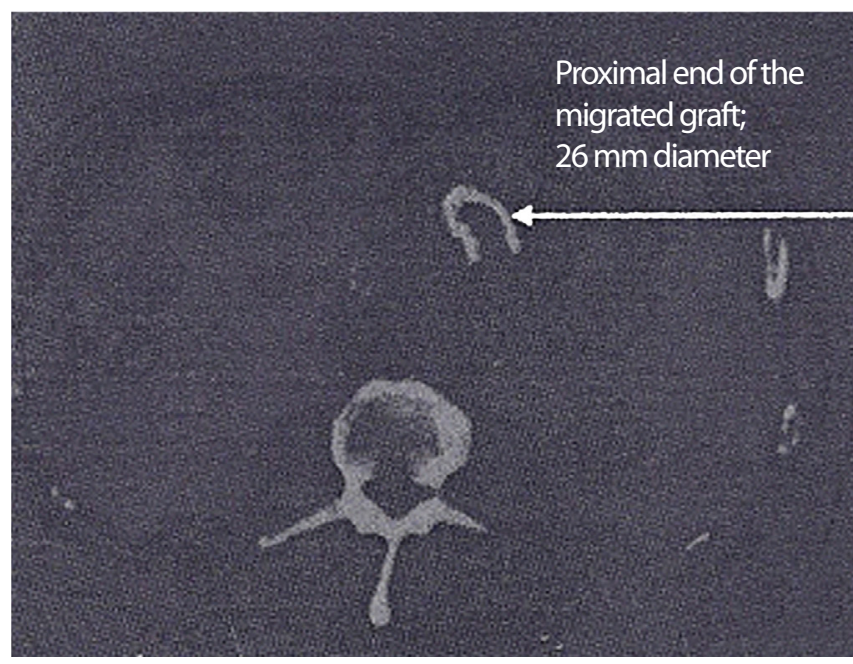

Fig. 1 - Pre-operative computed tomography (CT) scans: proximal end of migrated graft within the aneurysm sac.

should lie within the infrarenal neck and the two iliac limbs to extend itself from the main body into the iliac limbs of MG. All components were oversized by $10 \%$.

\section{Procedure}

The main body of SG (24F) was introduced from the right femoral artery and deployed just below the renal arteries. The distance between the distal end of SG and the proximal end of MG was $40 \mathrm{~mm}$. The left limb of SG's main body was cannulated by attaching the ipsilateral and contralateral magnets within the MG's main body. The contralateral magnet wire was then exchanged for a Lunderquist wire. A $17 \mathrm{~mm} \times 140 \mathrm{~mm}$ contralateral iliac limb was used to bridge the gap between SG and $M G$, landing into the left iliac limb of MG. An ipsilateral 17 $\mathrm{mm} \times 140 \mathrm{~mm}$ iliac limb was deployed from the right iliac limb of SG into the right limb of MG. Thus, a new modular endograft was inserted above and into the $M G$, thereby excluding the aneurysm sac from blood flow. Intraoperative angiogram didn't reveal endoleaks. Pedal pulses were palpable postoperatively. The duration of the procedure was 1 hour and 30 minutes.

The patient was discharged 72 hours later. CT scans at 6 weeks, 6 months and 18 months showed intact endograft components, absence of endoleaks and shrinkage of the aneurysm sac (Figure 2). He returned after 36 months with chest pain, hypotension and loss of sensation in both lower limbs. Investigations confirmed acute myocardial infarction (MI), lower limb ischemia and severe renal dysfunction. Ultrasound revealed absence of blood flow within the stent-graft. He was assessed as being too ill for any cardiological or vascular intervention. He demised within 48 hours of admission. It was unclear whether it was the lower limb ischemia or cardiac ischemia that initiated his current condition.

\section{DISCUSSION}

The Society for Vascular Surgery (SVS) define migration as the movement of the endograft by $10 \mathrm{~mm}$ relative to anatomical landmarks or any migration leading to symptoms or requiring

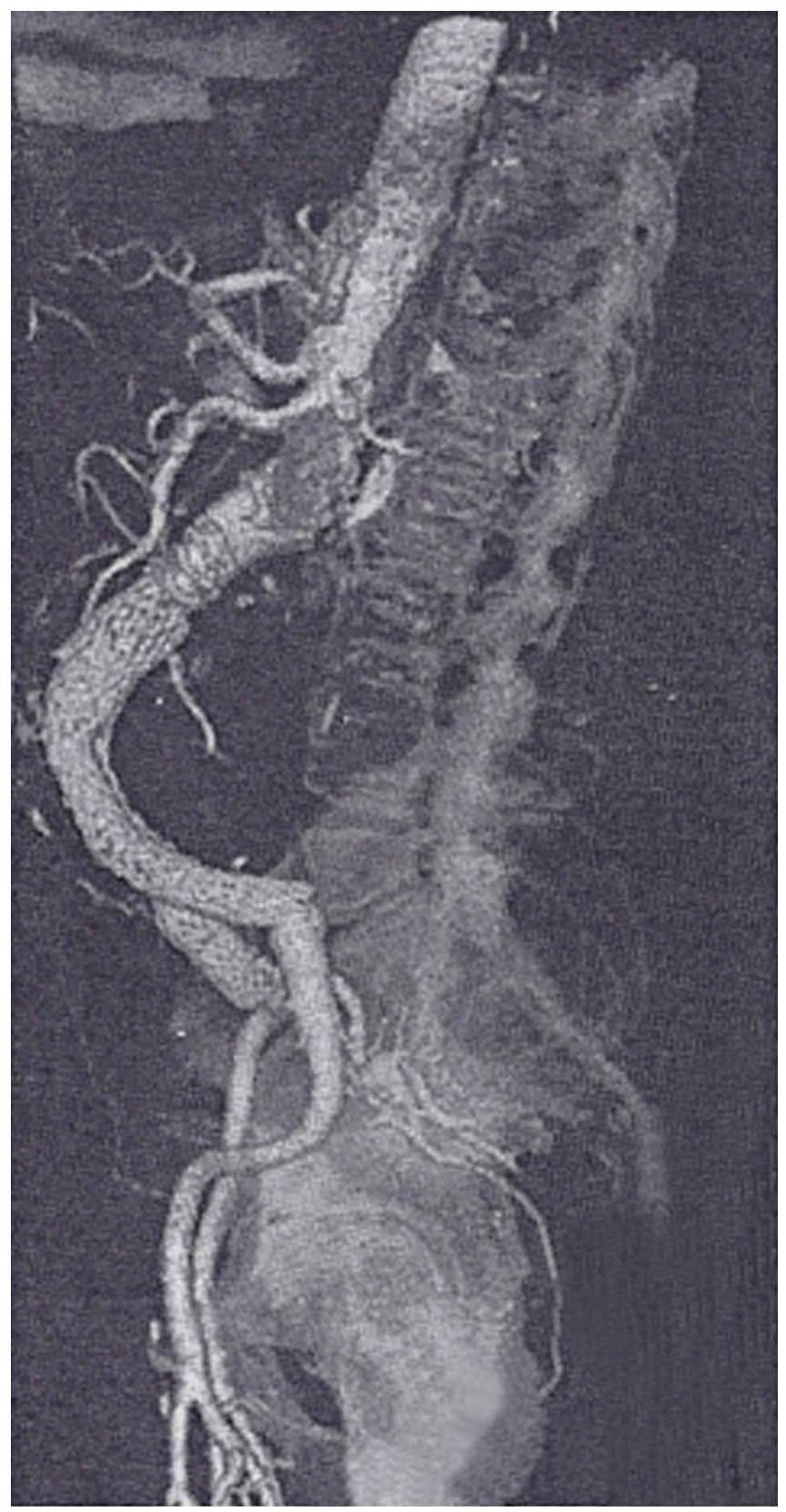

Fig. 2 - Six weeks follow-up computed tomography (CT) scan.

therapy ${ }^{[6]}$. There are no guidelines for treating complete migration ${ }^{[5]}$. Open repair is the recommended procedure of choice to treat complete migration.

In this case, due to inflammation, graft component incorporation and the prohibitive comorbidities, endovascular repair seemed to be a better option. The use of an extender cuff only was not an option because the graft had completely migrated. The AnacondaTM device was chosen. Its short main body allowed a "working length" to place two iliac extension limbs between SG and MG. The lower limb ischemia prior to his death could have been due to component occlusion, kinking or migration. Stent-graft thrombus secondary to MI and hypotension was also possible. In appropriate individuals, such 
"off-label" use of EVAR devices requires a stricter follow-up or even conversion to open repair at a later stage if the patient's general condition allows it.

Although EVAR relining by using a second infrarenal endograft seems to be a feasible alternative to immediate open surgery in high-risk patients, with a complete MG, other options need to be investigated. Customizing EVAR devices in this setting demands more investigation.

\section{Authors' roles \& responsibilities}

JP Substantial contributions to the conception or design of the work; final approval of the version to be published

$\mathrm{CY}$ Substantial contributions to the conception or design of the work; final approval of the version to be published

MO Substantial contributions to the conception or design of the work; final approval of the version to be published

MGV Substantial contributions to the conception or design of the work; final approval of the version to be published

\section{REFERENCES}

1. Greenhalgh RM, Brown LC, Kwong GP, Powell JT, Thompson SG, EVAR trial participants. Comparison of endovascular aneurysm repair with open repair in patients with abdominal aortic aneurysm (EVAR Trial 1), 30-day operative mortality results: randomised controlled trial. Lancet. 2004;364(9437):843-8.

2. Laheij RJ, Buth J, Harris PL, Moll FL, Stelter WJ, Verhoeven EL. Need for secondary interventions after endovascular repair of abdominal aortic aneurysms. Intermediate-term follow-up results of a European collaborative registry (EUROSTAR). Br J Surg. 2000;87(12):1666-73.

3. Buth J, Harris PL, van Merrewijk C, Fransen G. The significance and management of different types of endoleaks. Semin Vasc Surg. 2003;16(2):95-102.

4. Malas MB, Ohki T, Veith FJ, Chen T, Lipsitz EC, Shah AR, et al. Absence of proximal neck dilatation and graft migration after endovascular aneurysm repair with balloon-expandable stent-based endografts. J Vasc Surg. 2005;42(4):639-44.

5. Cao P Verzini F, Zannetti S, De Rango P, Parlani G, Lupattelli L, et al. Device migration after endoluminal abdominal aortic aneurysm repair: analysis of 113 cases with a minimum follow-up period of 2 years. J Vasc Surg. 2002;35(2):229-35.

6. Chaikof EL, Blankensteijn JD, Harris PL, White GH, Zarins CK, Bernhard VM, et al. Reporting standards for endovascular aortic aneurysm repair. J Vasc Surg. 2002;35(5):1048-60. 